Kansas State University Libraries

New Prairie Press

\title{
CHARACTERIZING THERMAL HYSTERESIS IN BODY TEMPERATURE FOR A HEAT STRESSED STEER
}

F. Yang

A. M. Parkhurst

D. A. Spiers

J. B. Gaughan

T. L. Mader

See next page for additional authors

Follow this and additional works at: https://newprairiepress.org/agstatconference

Part of the Agriculture Commons, and the Applied Statistics Commons

\section{(c) (1) 9(}

This work is licensed under a Creative Commons Attribution-Noncommercial-No Derivative Works 4.0 License.

\section{Recommended Citation}

Yang, F.; Parkhurst, A. M.; Spiers, D. A.; Gaughan, J. B.; Mader, T. L.; and Hahn, G. L. (2010). "CHARACTERIZING THERMAL HYSTERESIS IN BODY TEMPERATURE FOR A HEAT STRESSED STEER," Conference on Applied Statistics in Agriculture. https://doi.org/10.4148/2475-7772.1060

This is brought to you for free and open access by the Conferences at New Prairie Press. It has been accepted for inclusion in Conference on Applied Statistics in Agriculture by an authorized administrator of New Prairie Press. For more information, please contact cads@k-state.edu. 


\section{Author Information}

F. Yang, A. M. Parkhurst, D. A. Spiers, J. B. Gaughan, T. L. Mader, and G. L. Hahn 


\title{
CHARACTERIZING THERMAL HYSTERESIS IN BODY TEMPERATURE FOR A HEAT STRESSED STEER
}

\author{
F. Yang ${ }^{1}$, A. M. Parkhurst ${ }^{1}$, D. A. Spiers ${ }^{2}$, J. B. Gaughan ${ }^{3}$, T. L. Mader ${ }^{4}$ and G. L. Hahn ${ }^{5}$ \\ ${ }^{1}$ Department of Statistics, University of Nebraska - Lincoln \\ ${ }^{2}$ School of Animal Studies, University of Missouri - Columbia \\ ${ }^{3}$ School of Animal Studies, The University of Queensland, Gatton, Australia \\ ${ }^{4}$ Department of Animal Science, University of Nebraska at Lincoln \\ ${ }^{5}$ USDA-ARS U.S. Meat Animal Research Center, U.S. Department of Agriculture(Retired)
}

\begin{abstract}
Studies have shown that exposure of animals to a high ambient temperature environment poses serious threats to their health, performance and productivity. Above a certain threshold an animal's body temperature $(\mathrm{Tb})$ appears to be driven by the hot ambient temperature (Ta). For steers challenged by heat stress, the Tb-Ta relationship shows a dramatic increase in Tb per unit change of Ta and the dynamics of the Tb-Ta relationship follow a pattern which depends on whether Ta is increasing or decreasing. A delay becomes noticeable in a steer's thermo-regulatory response to Ta when Ta is controlled to be sinusoidal in the steer's heat stress chamber. In other words, Tb lags behind Ta. Consequently when plotted in a Tb-Ta phase diagram, a hysteresis effect appears in the form of a hysteresis loop, indicating the steer is thermally challenged. The hysteresis loop shows a rotated elliptical pattern which depends on the delay (or lag) between $\mathrm{Tb}$ and Ta. The angle of rotation of the hysteresis loop indicates the correlation (and lag) between $\mathrm{Tb}$ and Ta. The area of the elliptical hysteresis loop can be used to quantify the amount of heat stress during the period of thermal challenge. For example, results of a thermal challenge of $32 \pm 7^{\circ} \mathrm{C}$ applied to a Hereford steer showed, the delay is longer (4 hr lag) and ellipse is larger in an acute stage than in a chronic stage (3 hr lag). A greater delay (or lag) suggests more time is needed to dissipate the heat stress. This result suggests, steers in an acute stage require more energy to dissipate heat than steers in a chronic stage.
\end{abstract}

Key words: Delay-relay model, Ta-Tb phase diagrams, Thermo-regulatory response, Threshold, Sinusoidal input, Limit cyclic, Thermal ellipse, Hysteresis loop, Tb-Ta lags, Acute stage, Chronic stage, Energy dissipation, Ambient-body temperature correlation, Hereford steers.

\section{Introduction}

The phenomenon of hysteresis can be defined in terms of the relationship between the output of a system and the inputs. The three fundamental properties of the output from a hysteretic process are: memory, rate independence, and initial state of the process. 
Cyclic hysteresis describes the behavior of a hysteretic process in response to periodic variations of external variables. Thermal hysteresis is one of cyclic hysteresis. In animal science, when an animal is thermally challenged, physiological processes in the body become activated in order to dissipate the increasing heat load. Body temperature $(\mathrm{Tb})$ is one process that shows a dramatic response to heat stress (HS). Studies show Tb is essentially unresponsive to air temperature (Ta) until a threshold is reached and then there is a dramatic response to increasing air temperature (Hahn 1999; Mader et al. 2001). Parkhurst et al. (1999) present a model (called PET) and showed that cattle are thermally challenged by exposure to hot cyclic Ta in controlled HS studies. The PET model has been successfully used to fit Tb of beef cattle in thermally challenging environments (Parkhurst et al. 1999, 2002a, 2002b; Sheng et al. 2001).

In 2010, Parkhurst presented a simplified version of the PET model illustrating the dynamics when the delay is known for animals thermally challenged in an environmental chamber. Thus, Tb can be predicted by assuming a specific delay or lag. In the transitional stage (dashed line Fig 1), the animal's Tb increases at an exponential rate as Ta increases. After the transitional stage, the animal's Tb becomes entrained in a stable periodic limit cycle (solid line, Fig 1). It indicates that once an animal becomes entrained in a state of thermal equilibrium and a jolt occurs in the trajectory, Tb will return to the periodic hysteresis as time progresses, although possibly with a shift in delay as a result of acclimatization by the animal.

Fig 1. Tb-Ta phase diagram shows trajectory from transitional to thermal hysteresis

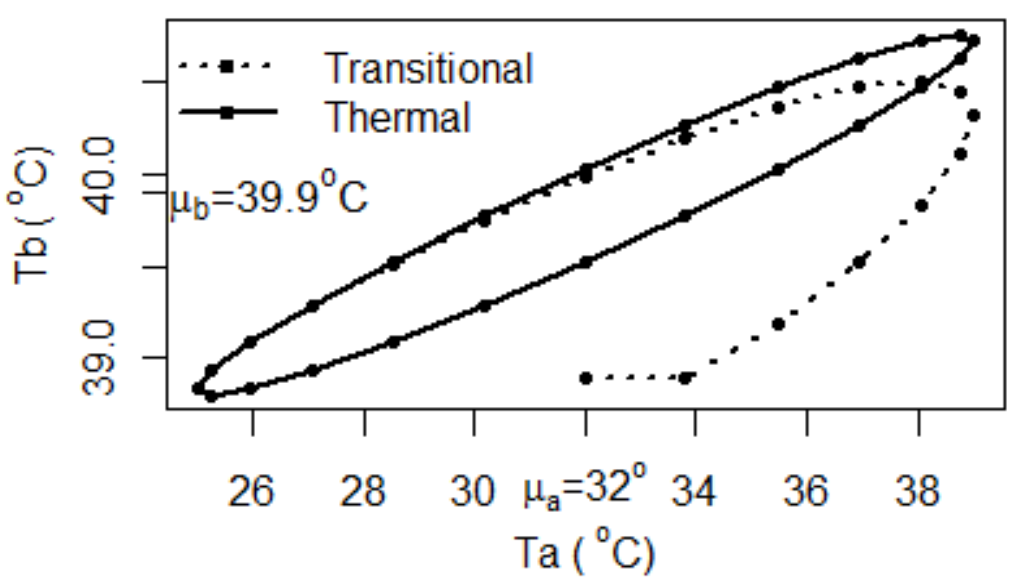

The elliptical loop formed during heat stress provides a way to quantify the amount of heat stress an animal experiences in a hot environment. Two features of the hysteretic ellipse, angle of rotation and area, help characterize the dynamics of heat stress. In this study, the angle of rotation of an ellipse is the angle between ellipse's major axis and the Ta (horizontal)-axis. It indicates the length of the time delay; i.e. how long Tb lags Ta. The lag can be estimated from the angle of rotation of an elliptical loop. In irreversible thermodynamics, the area inside the hysteresis loop equals the work done in one period or cycle (Brokate and Sprekels 1996). Thus, the area of the ellipse formed during thermal hysteresis is an indication of the animal's heat load. In other 
words, heat load can be assessed by estimating the area within the hysteresis loop.

$$
A_{H L}=\text { Area of ellipse }=\pi *\left(\frac{1}{2} \text { Major axis }\right) *\left(\frac{1}{2} \text { Minor axis }\right)
$$

A cross-correlation is a popular way to estimate lags and a linear regression can be used to predict $\mathrm{Tb}$. However, a simple linear model ignores the possibility of a trajectory. But more importantly, the ability to estimate the amount of heat load during thermal hysteresis is lost. Therefore, an appropriate model needs to provide estimates of both lag and heat load. One alternative is to model an ellipse. An elliptical model would provide a way to describe the lag and heat load characteristics of thermal hysteresis and make practical applications more germane.

The elliptical model can be used to examine differences in acute and chronic stages of HS. The premise is acute HS corresponds to a delay of $4 \mathrm{~h}$, while chronic HS corresponds to a delay of $3 \mathrm{~h}$ (Hahn et al. 1997). Results from Hahn also suggest that during the acute stage of HS, the animal dissipates more heat than in the chronic stage. Comparison of parameters estimated by the elliptical hysteresis model can be used to examine that premise.

The objectives of this study are to: 1) develop a procedure to fit a hysteretic ellipse; 2) illustrate the information lost by ignoring thermal hysteresis; and 3) present a practical application where parameter estimates from acute and chronic heat stress data are depicted.

\section{Materials and Methods}

\subsection{Statistical Methods}

When the Tb-Ta relationship is modeled as a simple linear regression, information about the delay and heat load is lost. To illustrate the loss of information, eigenvalues from the Tb-Ta correlation matrix are used to fit a unit ellipse to a system with homogeneous input and output variances. To exemplify fitting real data with heterogeneous input and output variances, a constrained least squares is performed.

\section{1.a. Correlation-Eigenvalue Method}

The formula for a unit ellipse is:

$$
\frac{\left(x-c_{x}\right)^{2}}{l_{x}^{2}}+\frac{\left(y-c_{y}\right)^{2}}{l_{y}^{2}}=1,
$$

where $\left(c_{x}, c_{y}\right)$ is the center of the ellipse, $l_{x}$ is the semi-length along the x-axis (Ta) and $l_{y}$ is the semi-length along the $\mathrm{y}$-axis $(\mathrm{Tb})$.

In the case of homogeneous variances, no information is lost when the covariance is standardized to form a correlation matrix and the angle of rotation is the correlation between $\mathrm{Tb}$ and Ta. If there is no delay, the angle is zero, and the correlation is $\cos (0)=1$. When there is no correlation, the angle is $90^{\circ}$, since $\cos (90)=0$. Eigenvalues from the correlation matrix give information about length and width of the ellipse. These are the ideas used in principle component analysis (Johnson and Wichern 2007). In this paper, the eigen-system from the correlation matrix between input and output 
variables is analyzed. Hence, the area of the unit ellipse is

$$
A_{\text {ellipse }}=\pi \sqrt{\lambda_{1} \lambda_{2}},
$$

with $\lambda_{1}>\lambda_{2}>0$ are eigenvalues from correlation matrix $\mathrm{R}$.

Then, (4) can be rewritten as a function of eigenvalues:

$$
\frac{\left(x-c_{x}\right)^{2}}{\lambda_{1}}+\frac{\left(y-c_{y}\right)^{2}}{\lambda_{2}}=1
$$

The correlation-eigenvalue method can be used to fit a unit ellipse to data in which input and output variables have homogeneous variance. The heat load during thermal hysteresis can be obtained from eigenvalues of the correlation matrix. This method provides a way to conceptualize the information lost by a simple linear regression model. However, it is not realistic to apply the correlation-eigenvalue method to real data when it has heterogeneous variances. The heterogeneity among variables may change the rotation of the ellipse. Then, the correlation-eigenvalue method will no longer give a reasonable representation of the data. To make the research results more applicable, a constrained ellipse-specific least squares method is performed.

\section{1.b. Ellipse-Specific Direct Least Squares Method}

Fitting of an ellipse is a classic problem in pattern recognition. Fitting generic conic shapes and implicit curves to real data points is an area of study in many fields such as engineering, biology and pharmaceutics. Many iterative methods have been explored on this issue. (Gander et al. 1994; Bookstein 1979; Pilu, Fitzgibbon and Fisher 1996; Fitzgibbon, Pilu and Fisher, 1999; Halir and Flusser 1998; O’Leary and Zsombor-Murray 2004) In the current study, the shape of the hysteresis loop is assumed to be elliptical. Thus, the ellipse-specific direct least squares algorithm of Pilu and Fitzgibbon (1996) was used to fit a hysterical ellipse to real data and estimates for both the rotation angle and amount of heat load were calculated.

Let $z_{i}=\left(x_{i}, y_{i}\right)$ be the $i^{\text {th }}$ observation of Ta and Tb over $i=1 \ldots \mathrm{n}$ time points. An ellipse can be written as a second order polynomial,

$$
\begin{aligned}
\begin{aligned}
F(\underset{\sim}{a}, \underset{\sim}{z}) & =\underset{\sim}{a z}=a_{1} x^{2}+a_{2} x y+a_{3} y^{2}+a_{4} x+a_{5} y+a_{6} \\
& =\left(\begin{array}{lll}
x & y & 1
\end{array}\right)\left(\begin{array}{ccc}
a_{1} & a_{2} / 2 & a_{4} / 2 \\
& a_{3} & a_{5} / 2 \\
& & a_{6}
\end{array}\right)\left(\begin{array}{l}
x \\
y \\
1
\end{array}\right)
\end{aligned} \\
\text { where } \underset{\sim}{a}=\left(\begin{array}{lllllllll}
a_{1} & a_{2} & a_{3} & a_{4} & a_{5} & a_{6}
\end{array}\right)^{\prime}, \underset{\sim}{z}=\left(\begin{array}{lllllll}
x^{2} & x y & y^{2} & x & y & 1
\end{array}\right)
\end{aligned}
$$

$F(\underset{\sim}{a} \underset{\sim}{z})=d$ is the algebraic distance of a point $(x, y)$ to the conic $F(\underset{\sim}{a}, \underset{\sim}{z})=0$.

To fit the ellipse, the algebraic distance is minimized over the set of $n$ points in the 
least squares sense; that is

$$
\hat{d}=\min _{a}\left\{\sum_{i=1}^{N} F\left(\underset{\sim}{a}, z_{i}\right)^{2}\right\}=\min _{a}\left\{\sum_{i=1}^{N}\left(a_{1} x_{i}^{2}+a_{2} x_{i} y_{i}+a_{3} y_{i}^{2}+a_{4} x_{i}+a_{5} y_{i}+a_{6}\right)^{2}\right\}
$$

To derive one and only one elliptical solution, a quadratic constraint $a_{2}^{2}-4 a_{1} a_{3}<0$ is added to (8).

Consequently, with the quadratic constraint (8) can be written as

$$
a^{t} S a-\lambda a^{t} C a=0 \text { for some } \lambda
$$

where $S=D^{t} D$ with $D=\left(\begin{array}{cccccc}x_{1}^{2} & x_{1} y_{1} & y_{1}^{2} & x_{1} & y_{1} & 1 \\ \ldots & \ldots & \ldots & \ldots & \ldots & \ldots \\ x_{n}^{2} & x_{n} y_{n} & y_{n}^{2} & x_{n} & y_{n} & 1\end{array}\right)$ ( D is called the design matrix,

O'Leary and Zsombor-Murray, 2004), and $C=\left(\begin{array}{cccccc}0 & 0 & -2 & 0 & 0 & 0 \\ 0 & 1 & 0 & 0 & 0 & 0 \\ -2 & 0 & 0 & 0 & 0 & 0 \\ 0 & 0 & 0 & 0 & 0 & 0 \\ 0 & 0 & 0 & 0 & 0 & 0 \\ 0 & 0 & 0 & 0 & 0 & 0\end{array}\right)$ such that $a_{2}^{2}-4 a_{1} a_{3}=a^{t} C a<0$.

Then (9) can be simplified to be a generalized eigenvalue problem; that $\dot{\mathrm{b}}$,

$$
S a=\lambda C a
$$

Since $S$ is positive definite (i.e. $a^{t} S a>0$ ) and $a^{t} C a<0, \lambda$ must be a single negative generalized eigenvalue. Therefore, the eigenvector corresponding to the negative eigenvalue in (10) is the unique elliptical solution to (8).

To solve the generalized eigen-problem (10), two cases need to be considered depending on the singularity of S. Datasets where the input and output are highly correlated (i.e. close to a straight line) may have eigenvalues close to zero. That is, the matrix S may be singular (non-invertible). Hence, the solution to the generalized eigen-problem (10) depends on the invertibility of $S$.

Case 1. If $S$ is invertible, then (10) can be written as

$$
\lambda^{-1} a=\left(S^{-1} C\right) a
$$

and (4) can be solved by singular value decomposition. The eigenvector corresponding to the negative eigenvalue in (11) is the same eigenvector in (10), and hence, is a solution of (9).

Case 2. If $\mathrm{S}$ is not invertible, then (10) has to be solved by the generalized 
eigen-problem. $S a=\lambda C a \rightarrow(S-\lambda C) a=0$. The aim is to find some $\lambda<0$ such that $\operatorname{det}|S-\lambda C|=0$. Since matrices $S$ and $C$ are known, it becomes a univariate root problem, which means $\lambda$ is the root of the function $\operatorname{det}|S-\lambda C|=0$. Thus, the corresponding generalized eigenvector in (10) is the kernel of $(S-\lambda C) a=0$.

Once the eigenvector is obtained, the real data can be written as a second order polynomial (7), and the ellipse is ready to be transferred into the canonical form. Suppose an ellipse along $u$ and $v$ axes is

$$
\left(\frac{u-c_{u}}{l_{u}}\right)^{2}+\left(\frac{v-c_{v}}{l_{v}}\right)^{2}-1=0,
$$

$\left(c_{u}, c_{v}\right)$ is the center point of the ellipse, $l_{u}$ and $l_{v}$ are the lengths of the half axes along the $u$ and $v$ axes, $(u, v)$ are the coordinates of the point rotated from the $x-y$ axes by the angle $\theta$, i.e., $\left\{\begin{array}{c}u=x \cos \theta+y \sin \theta \\ v=-x \sin \theta+y \cos \theta\end{array}\right.$.

Then, $\quad F(\underset{\sim}{a}, z)=\left(\frac{x \cos \theta+y \sin \theta-c_{u}}{l_{u}}\right)^{2}+\left(\frac{-x \sin \theta+y \cos \theta-c_{v}}{l_{v}}\right)^{2}-1$

Solving (13) for the five coefficients gives $\left(\begin{array}{l}a_{1}=\frac{\cos ^{2} \theta}{l_{u}{ }^{2}}+\frac{\sin ^{2} \theta}{l_{v}{ }^{2}} \\ a_{2}=\frac{2 \sin \theta \cos \theta}{l_{u}{ }^{2}}-\frac{2 \sin \theta \cos \theta^{2}}{l_{v}{ }^{2}} \\ a_{3}=\frac{\sin ^{2} \theta}{l_{u}{ }^{2}}+\frac{\cos ^{2} \theta}{l_{v}{ }^{2}} \\ a_{4}=-\frac{2 c_{u} \cos \theta}{l_{u}{ }^{2}}+\frac{2 c_{v} \sin \theta}{l_{v}{ }^{2}} \\ a_{5}=-\frac{2 c_{u} \sin \theta}{l_{u}{ }^{2}}-\frac{2 c_{v} \cos \theta}{l_{v}{ }^{2}} \\ a_{6}=\frac{c_{u}{ }^{2}}{l_{u}{ }^{2}}+\frac{c_{v}{ }^{2}}{l_{v}{ }^{2}}-1\end{array}\right)$

Hence, estimates for the angle, centroid, and semi-axes are:

$$
\begin{aligned}
& \hat{\theta}=\frac{1}{2}\left(\arctan \frac{a_{2}}{a_{1}-a_{3}}\right) \\
& \left(\begin{array}{l}
\hat{c}_{u} \\
\hat{c}_{v}
\end{array}\right)=-\frac{1}{2}\left(\begin{array}{cc}
a_{1} & a_{2} / 2 \\
a_{2} / 2 & a_{3}
\end{array}\right)^{-1}\left(\begin{array}{l}
a_{4} \\
a_{5}
\end{array}\right) \\
& \hat{l}_{u}=\sqrt{\frac{a_{1} \cos ^{2} \theta+a_{2} \sin \theta \cos \theta+a_{3} \sin ^{2} \theta}{\left(\begin{array}{ll}
c_{u} & c_{v}
\end{array}\right)\left(\begin{array}{cc}
a_{1} & a_{2} / 2 \\
a_{2} / 2 & a_{3}
\end{array}\right)\left(\begin{array}{l}
c_{u} \\
c_{v}
\end{array}\right)-a_{6}}} \\
& \hat{l}_{v}=\sqrt{\frac{a_{1} \sin ^{2} \theta-a_{2} \sin \theta \cos \theta+a_{3} \cos ^{2} \theta}{\left(\begin{array}{ll}
c_{u} & c_{v}
\end{array}\right)\left(\begin{array}{cc}
a_{1} & a_{2} / 2 \\
a_{2} / 2 & a_{3}
\end{array}\right)\left(\begin{array}{l}
c_{u} \\
c_{v}
\end{array}\right)-a_{6}}}
\end{aligned}
$$

The estimated area of the hysteresis loop is $\hat{A}_{\text {ellipse }}=\pi \hat{l}_{u}$. 
To predict $\mathrm{Tb}$ from Ta, a general parametric trigonometric form of an ellipse is required. Using the trigonometric form for $\mathrm{Ta}$ and $\mathrm{Tb}$ gives

$$
\begin{aligned}
& T a(t)=c_{u}+l_{u} \cos (t) \sin (\theta)+l_{v} \sin (t) \cos (\theta) \\
& T b(t)=c_{v}+l_{u} \cos (t) \cos (\theta)-l_{v} \sin (t) \sin (\theta)
\end{aligned}
$$

where $\left(c_{u}, c_{v}\right)$ is the center of the ellipse, $l_{u}$ and $l_{v}$ are the lengths of the semi-axes, $\theta$ is the rotation angle and $t$ varies from 0 to $2 \pi$. The input, Ta, and output, Tb, can be predicted by substituting the parameter estimates (14) into (15).

The ellipse-specific least squares method and simple linear model are compared by goodness-of-fit statistics such as MSE, Information Criteria (AIC and SBC), and prediction plots. The model with lower MSE, AIC and SBC, and no large residuals is preferred. The MSE for an ellipse was calculated using residuals from the algebraic distance (5) and $\mathrm{df}=\mathrm{n}-5$. All analyses in this study were done in R 2.11.0.

\subsection{Data: Simulated and Collected}

The implementations of the proposed methodologies methodology were is approached as proof-of-concept by applying the method to both simulated and collected data. Simulated data with homogeneous variances was is used to display the thermal dynamics and compare the results of fitting an elliptical model using correlation-eigenvalues to the results from a simple linear regression. Real data was fit using generalized eigenvalues to demonstrate the ellipse-specific direct least squares algorithm.

\section{2.a. Simulated data}

Ta was simulated in a sinusoidal pattern with mean 32, amplitude 1 , period $24 \mathrm{~h}$, and no error. Tb was simulated in the sinusoidal pattern with the same amplitude, period, and no error, but with a different mean 39.9, and lags at $0,1,2,3,4,6 \mathrm{~h}$ from the Ta.

\section{2.b Collected Data}

Data from one ad-lib-fed Hereford steer was randomly chosen from two steers in a controlled environment chamber. In an effort to emulate daily patterns of temperature behavior, the steers in the chamber were housed in a sinusoidal Ta regime. Tympanic temperatures were recorded on a one hour basis from 8:00am until the next day's 7:00am for fifteen days. Environmental conditions during the first 192 hours were thermoneutral cyclic $\left(10^{\circ} \mathrm{C} \pm 7^{\circ} \mathrm{C}\right)$. At hour 192 , conditions were elevated to hot cyclic $\left(28^{\circ} \mathrm{C} \pm 7^{\circ} \mathrm{C}\right)$. In this study, the Tb-Ta for two days under hot cyclic conditions corresponding to acute stage (day 10) and chronic stage (day 14) were examined.

\section{Results and Discussion}

\subsection{Correlation-Eigenvalues from Simulated Data}

In order to illustrate the advantages of an elliptical model, the correlation-eigenvalue method was applied to simulated data with homogeneous variances. The Tb-Ta phase diagrams provide visualizations of the thermal hysteresis loop (unit ellipse) for six 
delays (0, 1, 2, 3, 4 and $6 \mathrm{~h}$ ) corresponding to periods of $\quad 0$ to $\frac{1}{1 / 4}$ of a $24 \mathrm{~h}$, Fig 2.

The shapes of the ellipses fit by the correlation-eigenvalue method changed from a straight line to a circle as the length of the delay increased from 0 to 1/4 period. Hence, heat accumulation, in terms of area of the unit ellipse (hysteresis loop), increases from 0 to $\pi$ as the length of delay increases. Notice the linear regression (dashed line) becomes less and less plausible as a model for the data as hysteresis increases.

Fig 2. Simulated Hysteresis Loop (Unit Ellipse) Fit By Correlation-Eigenvalue Method (Solid Line) And Simple Linear Regression (Dashed Line).
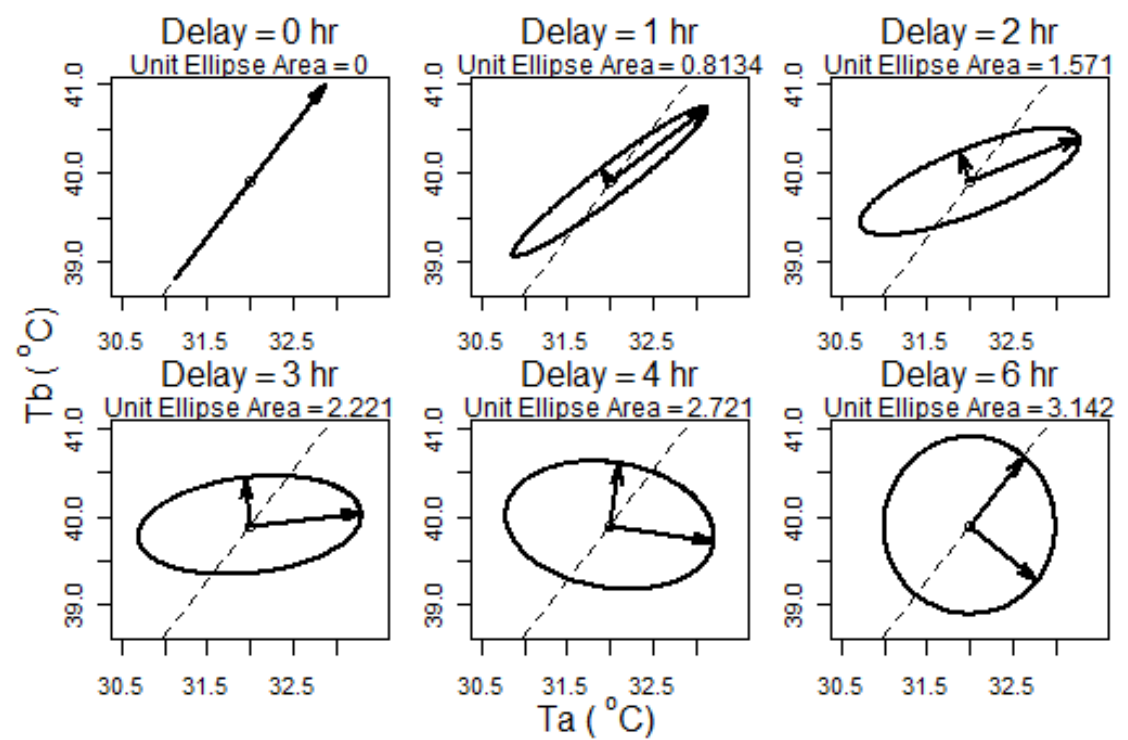

Table 1 further explores the loss of information as the delay increases. The correlation between $\mathrm{Tb}$ and $\mathrm{Ta}$ (cos(D egrees)) deteriorates, as does the proportion of total variation accounted for by the regression model (R-square). Estimates of the delay in $\mathrm{Tb}$ in response to Ta and the area of the unit ellipse were calculated using the correlation-eigenvalue method. The area of the hysteresis loop (unit ellipse) ranges from 0 to a unit circle.

Table 1. Comparison of Tb-Ta Correlation, R-square and Unit Ellipse Area for Different Delays.

\begin{tabular}{|c|c|c|c|c|c|c|}
\hline \multirow{2}{*}{ 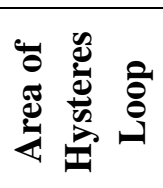 } & \multicolumn{3}{|c|}{$\begin{array}{c}\text { DELAY } \\
\text { for } \mathrm{Tb} \text { in response to } \mathrm{Ta}\end{array}$} & \multirow{2}{*}{$\begin{array}{c}\text { Tb-Ta } \\
\text { CORRELATION } \\
\text { Cos(Degrees) }\end{array}$} & \multirow{2}{*}{ R-square } & \multirow{2}{*}{$\begin{array}{l}\text { Unit } \\
\text { Ellipse } \\
\text { Area }\end{array}$} \\
\hline & Hour & Period & Degrees & & & \\
\hline \multirow{6}{*}{$\begin{array}{c}0 \\
\text { Small } \\
\mid \\
\mid \\
\text { Large }\end{array}$} & 0 & 0 & 0 & +1 & 1 & 0 \\
\hline & 1 & $1 / 24$ & 15 & .9659 & .9330 & 0.8134 \\
\hline & 2 & $1 / 12$ & 30 & .8660 & .7500 & 1.5709 \\
\hline & 3 & $1 / 8$ & 45 & .7071 & .5000 & 2.2215 \\
\hline & 4 & $1 / 6$ & 60 & .5 & .2500 & 2.7207 \\
\hline & 6 & $1 / 4$ & 90 & 0 & 0 & 3.1416 \\
\hline
\end{tabular}




\subsection{Estimating Thermal Hysteresis Characteristics from Collected Data}

Experimental data from acute and chronic stages of HS for one ad-lib-fed Hereford steer housed in a sinusoidally controlled $\left(28^{\circ} \mathrm{C} \pm 7^{\circ} \mathrm{C}\right)$ HS chamber were fit by the ellipse-specific direct least-squares method. The results from the ellipse-specific and simple linear regression models are compared in Fig 3. Both plots show that predictions from the ellipse-specific least squares method have smaller departures from the observed data than the linear regression method.

Fig 3. Predicted Tb-Ta from Ellipse-specific Hysteresis Model (solid line) and Simple Linear Regression (dashed line) Compared to Data Points (open circles)
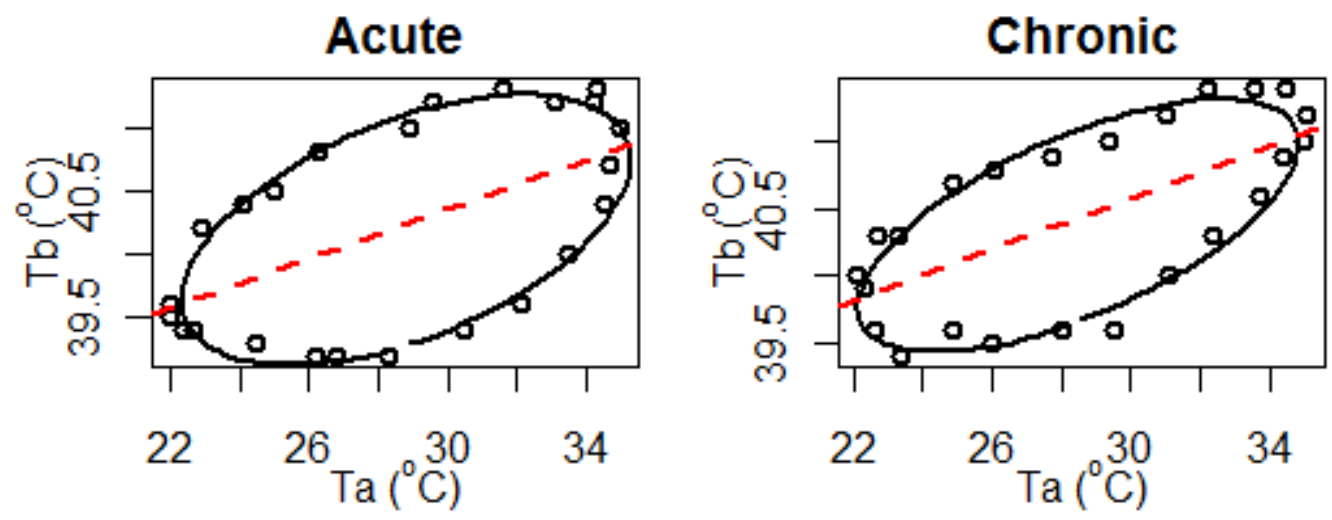

Statistics from both the ellipse-specific and linear regression models are summarized in Table 2. When compared to the linear regression model, the goodness-of-fit statistics (MSE, AIC and SBC) from the ellipse-specific model were much smaller in both stages. The results indicate an ellipse-specific model is significantly better than a linear regression model.

Table 2. Estimates of Thermal Characteristics and Goodness-of-Fit Statistics for Acute and Chronic Stages.

\begin{tabular}{|c|c|c|c|}
\hline \multicolumn{2}{|c|}{} & ACUTE & CHRONIC \\
\hline \multicolumn{2}{|c|}{ Angle of Rotation $\left({ }^{\circ}\right)$} & 5 & 5.63 \\
\hline \multicolumn{2}{|c|}{ Lag (h) from CCF } & 4 & 3 \\
\hline \multicolumn{2}{|c|}{ Lag from Ellipse (h) } & 3.84 & 3.36 \\
\hline \multicolumn{2}{|c|}{ Semi-Major Axis $\left({ }^{\circ} \mathrm{C}\right)$} & 6.5 & 6.4 \\
\hline \multicolumn{2}{|c|}{ Semi-Minor Axis $\left({ }^{\circ} \mathrm{C}\right)$} & 0.91 & 0.72 \\
\hline Area: Heat Accumulation $\left({ }^{\circ} \mathrm{C}^{2}\right)$ & 18.47 & 14.41 \\
\hline \multirow{2}{*}{ MSE } & Ellipse & $2.07 \mathrm{e}-29$ & $8.25 \mathrm{e}-29$ \\
\cline { 2 - 4 } & Regression & 0.44 & 0.28 \\
\hline AIC & Ellipse & 2.11 & 1.81 \\
\cline { 2 - 4 } & Regression & 52.47 & 41.11 \\
\hline SBC & Ellipse & 1.35 & 1.05 \\
\cline { 2 - 4 } & Regression & 56.01 & 44.65 \\
\hline
\end{tabular}




\subsection{Comparing Thermal Characteristics of Acute and Chronic Heat Stress Stages}

Table 2 shows that the angle of rotation was $5.00^{\circ}$ in an acute stage and was $5.63^{\circ}$ in a chronic stage. The estimated lag from CCF in the acute stage was $4 \mathrm{~h}$, which was larger than lag $3 \mathrm{~h}$ in chronic stage. The estimated lag from ellipse-specific model was $3.8 \mathrm{~h}$ in the acute stage and was $3.4 \mathrm{~h}$ in the chronic stage. When rounded to the nearest whole number lags estimated from ellipse-specific model agreed with lags estimated from CCF in both stages. The ellipse estimated for the acute stage had a semi-major axis of length $6.5^{\circ} \mathrm{C}$, a semi-minor axis of length $0.91{ }^{\circ} \mathrm{C}$ and area of $18.4{ }^{\circ} \mathrm{C}^{2}$; while for the chronic stage, the length of the semi-major axis was $6.4^{\circ} \mathrm{C}$, the length of the semi-minor axis was 0.72 and area was $14.41^{\circ} \mathrm{C}^{2}$. In this study, the heat accumulation in the acute stage $\left(18.5^{\circ} \mathrm{C}^{2}\right)$ was $4{ }^{\circ} \mathrm{C}^{2}$ more than in the chronic stage $\left(14.4{ }^{\circ} \mathrm{C}^{2}\right)$. The results from the ellipse-specific model agree with those stated by Hahn, et.al (1997) and suggest that animals dissipate more energy in acute stage HS than in chronic stage HS.

\section{Conclusion}

The ellipse-specific least squares method is a more accurate way to estimate the lag between $\mathrm{Tb}$ and $\mathrm{Tb}$ than estimating cross-correlation coefficients. Moreover, an ellipse provides a good model for the thermal hysteresis loop. The ellipse-specific direct least squares method allows us to fit a rotated ellipse to real data and to calculate the angle of rotation, length of the axes and area of the ellipse. The size of the thermal hysteresis loop depends on the size of the delay in $\mathrm{Tb}$, which in turn depends on the environment and how quickly an animal reacts to the change in the environment. Once an animal becomes entrained in a thermal hysteresis loop, they may experience acute or chronic HS until physiological or environmental conditions change. Hence, the size of the thermal hysteresis loop represents the amount of energy dissipated by an animal during a $24 \mathrm{~h}$ period.

\section{Summary}

Health of animals is compromised if they were exposed to a thermally challenging environment. During heat stress, there is a noticeable delay in the Tb-Ta relationship when an animal is exposed to a controlled hot sinusoidal thermal environment. The Tb-Ta phase diagram shows a hysteresis effect in the form of a rotated elliptical hysteresis loop. The angle of rotation of the hysteresis loop indicates the amount of lag between $\mathrm{Ta}$ and $\mathrm{Tb}$; the area of the hysteresis loop indicates the amount of heat accumulated during a thermal challenge. To estimate the lag and heat loss, two methods can be used. The correlation-eigenvalue method works well in a datasets with homogeneous variances, while the ellipse-specific direct least-squares method provides a more general approach to include datasets with heterogeneous variances. To demonstrate how the Tb-Ta relationship changes in the presence of hysteresis, an elliptical model was fit to simulated homogeneous data by solving the correlation-eigenvalue system. Results from the simulated data show how the area of the unit ellipse increases as Tb increasingly lags Ta. Additionally, these results call attention to the information lost by ignoring hysteresis and merely fitting a simple 
linear regression. To demonstrate a practical application, the ellipse-specific direct least-squares method was applied to two stages of HS for an ad-lib-fed Hereford steer housed in a sinusoidally controlled $\left(28^{\circ} \mathrm{C} \pm 7^{\circ} \mathrm{C}\right) \mathrm{HS}$ chamber. The animal in an acute stage had a longer lag (3.8 $4 \mathrm{~h}$ ) than it did in a chronic stage (3.4 $3 \mathrm{~h}$ lag). The area of the fitted ellipse in the acute stage was larger than the area of the fitted ellipse in the chronic state. Depicting the thermal dynamics of an animal is a complicated process. The ellipse-specific direct least-squares method allows us to further the characterize the dynamic process by providing a way to quantify the heat load as well as a more accurate estimate the Tb-Ta lag.

\section{References}

Bookstein, F.L. 1979. Fitting conic sections to scattered data. Computer Graphics and Image Processing 9:56-71.

Brokate, M. and J. Sprekels. 1996. Hysteresis and phase transitions. New York: Springer.

Fitzgibbon, A.W., M. Pilu and R.B. Fischer. 1999. Direct least squares fitting of ellipses. IEEE Transactions on Pattern Analysis and Machine Intelligence 21(5):476-480.

Gander, W., G.H. Golub and R. Strebel. 1994. Least-squares fitting of circles and ellipses. BIT Numerical Mathematics 43:558-578.

Hahn, G.L. 1999. Dynamic responses of cattle to thermal heat loads. Journal of Animal Science 77(Supplement 2):10-20.

Hahn, G.L., A.M. Parkhurst and J.B. Gaughan. 1997. Cattle respiration rate as a function of ambient temperature. ASAE Mid-Central Conf. No.MC97-121. American Society of Agricultural Engineers, St Joseph, MI

Halir, R. and J. Flusser. 1998. Numerically stable direct least squares fitting of ellipses. Proc. WSCG'98. The 6th International Conference in Central Europe on Computer Graphics and Visualization'98 in co-operation with EUROGRAPHICS and IFIP. Czech Republic. 125-132.

Johnson, R.A. and D.W. Wichern. 2007. In: Applied multivariate statistical analysis. 6th Edition ed. New Jersey: Prentice Hall. 49-65,111-129,149-155 p.

Lapshin, R.V. 1995. Analytical model for the approximation of hysteresis loop and its application to the scanning tunneling microscope. Review of Scientific Instruments 66(9):4718-4730.

Mader, T.L., S.M. Holt, J.B. Gaughan, G.L. Hahn, M.S. Davis, A.M. Parkhurst, D.E. Spiers. 2001. Heat load management for feedlot cattle. Proc. 6th International Livestock Environment Symposium. American Society of Agricultural Engineers, St. Joseph, MI. 147-153

O’Leary, P. and P. Zsombor-Murray. 2004. Direct and specific least-square fitting of hyperbolæ and ellipses. Journal of Electronic Imaging 13(3):492-503.

Parkhurst A.M., G.L. Hahn, K.M. Eskridge, D.A. Travnicek, H.D. Liu. 1999. Predicting body temperature of cattle during stages of exposure to controlled hot 
cyclic air temperature using metrics with specified delays. Proc. 15th International Congress of Biometeorology \& International Conference on Urban Climatology, 274-280, CD Rom ICB13.2

Parkhurst, A.M., D.A. Spiers, T.L. Mader, and G.L.Hahn, 2002a. What is the Definition of Heat Stress Threshold? Proc. 15th Conference on Biometeorology and Aerobiology co-sponsored by International Society of Biometeorology and the American Meteorological Society, Kansas City MO. 162-165

Parkhurst, A.M., D.A. Spiers, T.L. Mader, and G.L. Hahn, 2002b. Spline models for estimating heat stress thresholds in cattle. Proc. 14th Annual Kansas State University Conference on Applied Statistics in Agriculture. Kansas State Univ. Manhattan, KS. 137-148

Parkhurst, A.M. 2010. Model for understanding thermal hysteresis during heat stress: A matter of direction. International Journal of Biometeorology. Springer. Published online: http://www.springerlink.com/content/gp2x266nx7879503/.

Pilu, M., A.W. Fitzgibbon and R.B. Fisher. 1996. Ellipse specific direct least squares fitting. Proc. IEEE international Conference on Image Processing. Los Alamitos, CA: IEEE Computer Society Press, 3:599-602

R Development Core Team, 2009. R: A Language and Environment for Statistical Computing. R Foundation for Statistical Computing, Vienna, Austria. ISBN 3-900051-07-0, URL http://www.R-project.org

Sheng, F., A.M. Parkhurst, K. Eskridge, D. Travnicek, D. Spiers, G.L. Hahn and T.L. Mader. 2001. Assessing refinements in modeling sinusoidal conditions used to drive cattle body temperature. Proc. 13th Annual Kansas State University Conference on Applied Statistics in Agriculture. Kansas State Univ. Manhattan, KS. 299-307 